\title{
La interpretación en las organizaciones internacionales. Investigación, formación y práctica
}

\section{PREFACIO}

\section{Mariachiara RusSO e Icíar ALONSO-ARAGUÁS}

(GIR Alfaqueque, USAL)

Università di Bologna/Forli / Universidad de Salamanca

mariachiara.russo@unibo.it / itziar@usal.es

Los intérpretes profesionales tienen la posibilidad de ejercer su trabajo como autónomos en el mercado privado o como funcionarios de instituciones nacionales o internacionales. También pueden optar por una combinación de ambas opciones ejerciendo como autónomos para las organizaciones internacionales.

Cada una de estas elecciones es el reflejo de las aspiraciones y necesidades de cada uno, de un estilo de vida ligado a una práctica profesional que despierta admiración, pues requiere una alta cualificación y además facilita la comunicación entre las personas. Sin embargo, algunas situaciones en las que hoy deben trabajar los intérpretes generan también cierta inquietud: sucede en condiciones laborales especialmente complejas durante y alrededor del proceso de comunicación (Kalina 2005: 778), como los contextos multilingües donde se usa (o más bien se abusa de) una lingua franca, o en situaciones que pueden llegar a poner su vida en peligro, como la interpretación en zonas de conflicto.

El objetivo de este número monográfico es ofrecer una amplia panorámica sobre la práctica de la interpretación en las organizaciones internacionales desde la perspectiva 
de quienes trabajan o han trabajado en ellas como intérpretes profesionales, unida a la experiencia de numerosos intérpretes consultados en dos valiosas encuestas originales y a los resultados de varios estudios elaborados por prestigiosos investigadores del área.

Este volumen abarca el recorrido completo de un intérprete que se plantee ejercer en este tipo de situaciones comunicativas mediadas por intérpretes: desde los cursos de formación en interpretación, pasando por el reto que supone el proceso de selección mediante exámenes de acceso, hasta el amplio abanico de instituciones multilingües de prestigio que contratan a intérpretes profesionales, con especial atención a la Organización de las Naciones Unidas (ONU), la Organización para la Cooperación y el Desarrollo Económicos (OCDE) y la Unión Europea (UE). En los próximos meses verá la luz un segundo número monográfico de CLINA sobre el mismo tema, dedicado íntegramente a la UE.

En el primer capítulo, Training Interpretation Students at Middlebury Institute of International Studies at Monterey (MIIS) to Work for International Organizations (La formación de los estudiantes de interpretación en el Middlebury Institute of International Studies de Monterrey (MIIS) para trabajar en organizaciones internacionales), Leire Carbonell-Agüero presenta el ejemplo paradigmático de un prestigioso centro de formación de traductores e intérpretes. La autora expone el planteamiento y las señas de identidad del plan de estudios de su institución, cuyos rasgos fundamentales comparten evidentemente los programas de los másteres acreditados en formación de intérpretes. Destaca sobre todo su enfoque del aprendizaje centrado en el alumno, junto con la indiscutible ventaja de contar como docentes con intérpretes profesionales en activo que acercan a los estudiantes los temas y los criterios de calidad del mercado. El centro dispone además de un programa tutelado de prácticas curriculares en situaciones reales de interpretación, lo que a todas luces constituye un excelente ejemplo de buenas prácticas. Esta última característica, presente también en otros centros de formación superior de todo el mundo, es la más significativa y constituye sin duda el mejor catalizador posible para transformar lo adquirido por el alumno en el aula en destrezas de interpretación capaces de responder a los retos que plantea la práctica profesional en la vida real. De este modo, los estudiantes descubren el placer de actuar como si fueran intérpretes profesionales, lo que a su vez les anima a intentar alcanzar altos niveles de calidad. Insistiendo en la conveniencia de que las escuelas de formación doten a los estudiantes de las herramientas precisas para que puedan superar las pruebas de acceso a las organizaciones internacionales y trabajar como autónomos o como funcionarios, Carbonell-Agüero pone el acento en los principales retos a los que se enfrentan hoy los candidatos. En lo que atañe a la $\mathrm{ONU}$, se hace eco de la cuestión suscitada por Marie Diur cuando se pregunta «por qué el índice de éxito en las oposiciones para intérpretes de plantilla de las Naciones Unidas (The United Nations' Language Competitive Examination) suele ser medio o

Mariachiara RUSSO e Icíar ALONSO-ARAGUÁS

La interpretación en las organizaciones internacionales. Investigación, formación y práctica
CLINA

vol. 3-2, December 2017, 7-17

elSSN: 2444-1961

(C) Ediciones Universidad de Salamanca - CC BY-NC-ND 
bajo», y establece así un «diálogo virtual» con las autoras de la segunda contribución que figura en este volumen: Admission exams in international organisations: The United Nations' Language Competitive Examination (LCE) [Los exámenes de admisión en las organizaciones internacionales: la oposición para intérpretes de las Naciones Unidas (LCE)], de Lucía Ruiz Rosendo y Marie Diur.

En su detallada presentación sobre la estructura de las Naciones Unidas, las características de este tipo de oposición y la preparación requerida, Ruiz Rosendo y Diur ofrecen una valiosa orientación para todos aquellos intérpretes que deseen trabajar como intérpretes para la ONU. Las autoras consideran que estos exámenes reflejan los tres desafíos principales que afrontan a diario los intérpretes de la organización, según se desprende de su encuesta dirigida a los intérpretes de plantilla de las 6 cabinas de interpretación: la velocidad, la temática específica de la ONU y los acentos. En cuanto a la velocidad, las autoras apuntan un dato muy interesante: la velocidad media a la que se pronuncian los discursos en las Naciones Unidas es de 161 ppm, y de 160 ppm para los discursos cuya lengua de partida (LP) es el inglés. En el Parlamento Europeo los intérpretes de simultánea trabajan bajo una presión similar, pues se ha calculado que la velocidad media de los discursos en las sesiones plenarias es de 130160 ppm, y de 157 ppm para los discursos en inglés como LP (Sandrelli et al. 2010, Iglesias Fernández 2010). Al igual que nuestra anterior autora, Lucía Ruiz Rosendo y Marie Diur insisten en la necesidad de que las escuelas de formación en interpretación proporcionen a los estudiantes las destrezas necesarias para superar el examen de acceso y tratan de ayudar a los candidatos a anticiparse a las dificultades que conlleva dicha prueba.

Las tres contribuciones siguientes se centran en las dificultades mencionadas por los intérpretes profesionales de varias organizaciones internacionales multilingües: Jesús Baigorri-Jalón y Críspulo Travieso-Rodríguez se refieren a las Naciones Unidas en sus sedes de Nueva York, Ginebra y Viena; Kilian Seeber a la Unión Europea; y Clare Donovan a la OCDE, radicada en París.

Jesús Baigorri-Jalón y Críspulo Travieso-Rodríguez, en su capítulo titulado Interpreting at the United Nations: the impact of external variables. The Interpreters' View (La interpretación en las Naciones Unidas: el impacto de variables externas. El punto de vista de los intérpretes), presentan los resultados de una encuesta dirigida a intérpretes funcionarios de la ONU donde les preguntaban cómo habían influido en su ejercicio profesional los cambios experimentados en las dos últimas décadas en sus condiciones laborales. Sus respuestas ilustran de forma muy nítida cómo a lo largo de los años las tecnologías, el uso generalizado del relé, el inglés como lingua franca, los temas de trabajo, los acentos, la lectura de los discursos y, sobre todo, la elevada velocidad con la que se presentan han ido modificando su carga de trabajo.

Estos tres últimos elementos -velocidad, acentos y naturaleza escrita de los discursos leídos- ocupan también los primeros lugares en la lista de obstáculos con

Mariachiara RUSSO e Icíar ALONSO-ARAGUÁS

La interpretación en las organizaciones internacionales. Investigación, formación y práctica 
Ios que han de lidiar los intérpretes en el Parlamento Europeo y que Kilian Seeber ha analizado con todo lujo de detalles en su trabajo Interpreting at the European Institutions: Faster, higher, stronger, (La interpretación en las instituciones europeas: más rápido, más alto, más fuerte). Su enfoque se centra en el procesamiento lingüístico en general, y en la interpretación simultánea de conferencias en particular. Además de ofrecernos una minuciosa descripción de las condiciones de trabajo y de las características de los encargos y reuniones con interpretación en la UE, con especial referencia a los tres elementos señalados, el autor se detiene en cada uno de ellos examinando en profundidad las implicaciones lingüísticas y cognitivas de la comprensión y de la producción, para ocuparse luego de sus efectos en el proceso de interpretación simultánea. Con argumentos apoyados en la evidencia y en la bibliografía especializada, destaca las dificultades a las que se enfrentan los intérpretes de simultánea, sobre todo cuando esos tres retos mencionados se combinan entre sí como viene ocurriendo cada vez más a menudo en las organizaciones internacionales, y aboga por soluciones tecnológicas y estratégicas que respalden el trabajo a veces desalentador de los intérpretes. Esta demanda de ayuda cobra todavía más sentido a la luz de los últimos desarrollos de las TIC, que permiten la interpretación asistida por ordenador (CAl, por sus siglas en inglés) con aplicaciones capaces de optimizar el tiempo y los recursos. Así sucede, por ejemplo, con la integración de la CAl y de los programas de Reconocimiento Automático del Habla para buscar automáticamente el léxico especializado y situar en la pantalla posibles traducciones en el idioma meta junto a otros elementos potencialmente problemáticos como nombres propios, acrónimos o cifras (Fantinuoli 2017).

Nuestra siguiente autora, Clare Donovan, se hace eco de esta misma necesidad de disponer de nuevos recursos de comunicación y de tecnologías de la información para estar a la altura de los cambios experimentados en los modos de presentación de discursos y en el formato de las reuniones. En su capítulo titulado The Place of the Interpreter and Interpreting in an Institutional Setting (El lugar del intérprete y de la interpretación en un entorno institucional), Donovan nos traslada a un ámbito distinto de intercambio multilateral como es la Organización para la Cooperación y el Desarrollo Económicos (OCDE), con sede en París. El trabajo se abre con una cuidada presentación sobre el funcionamiento de la institución, los requisitos de los intérpretes y las tareas que estos llevan a cabo. Sin perder de vista la evolución histórica de la OCDE, la autora desvela a continuación los distintos cambios que han afectado a la práctica de la interpretación: el uso generalizado de la interpretación simultánea y del inglés como lingua franca, y la interpretación y participación remota en las conferencias, con el consiguiente impacto en las percepciones y tareas de los intérpretes. Una vez más, la elevada velocidad de los discursos originales emerge aquí como uno de los parámetros con mayor incidencia en el buen desempeño de la interpretación y, en líneas generales, en la comunicación.

Mariachiara RUSSO e Icíar ALONSO-ARAGUÁS

La interpretación en las organizaciones internacionales. Investigación, formación y práctica 
El colofón a estas contribuciones corre a cargo de Marija Todorova, Interpreting at the Border: "Shuttle interpreting" for the UNHCR (Interpretar en la frontera: «Interpretación exprés» para el ACNUR). Con ella abandonamos la comodidad de las salas de conferencias equipadas con cabinas de interpretación para conocer las misiones sobre el terreno, donde nos enfrentamos a numerosos retos y a veces de distinta naturaleza. En este escenario el intérprete adquiere mayor visibilidad y ejerce de lleno su papel como mediador. Todorova nos presenta dos estudios de caso: uno sobre la experiencia de la propia autora en Macedonia y Kosovo y el otro, sobre la de una de sus colegas en Macedonia. Como ella misma señala, la interpretación en zonas de conflicto sitúa bajo un nuevo prisma los principios éticos y deontológicos tradicionales en la profesión y saca a la luz el papel del intérprete en la defensa de las personas en situación vulnerable.

Para concluir, cabe señalar que los trabajos que componen este número especial brindan una completa panorámica sobre el trabajo de los intérpretes en organizaciones internacionales multilingües desde diferentes perspectivas: la formación, los procesos de selección, y la práctica profesional en un amplio abanico de ámbitos de trabajo y de desafíos inherentes a cada uno de ellos.

Confiamos, pues, en que tanto los estudiantes, como los formadores y los intérpretes encuentren en estas páginas un motivo de estímulo para su profesión y también para futuras investigaciones.

Las editoras

\section{REFERENCIAS BIBLIOGRÁFICAS}

IGLESIAS FERNÁNDEZ, Emilia. 2010. «Speaker fast tempo and its effect on interpreter performance: A pilot study of a multilingual interpreting corpus». International Journal of Translation 22 (12): 205-228.

FANTINUOLI, Claudio. 2017. «Speech Recognition in the Interpreter Workstation». Proceedings of the Translating and the Computer 39. Accessed November 28 2017. https://www. researchgate.net/publication/321137853_Speech_Recognition_in_the_Interpreter_ Workstation

KALINA, Sylvia. 2005. "Quality Assurance for Interpreting Processes», Meta 50 (2): 768-784. doi:10.7202/011017ar

SANDRELLI, Annalisa, BENDAZZOLI, Claudio and Mariachiara RuSSO. 2010. «European Parliament Interpreting Corpus (EPIC): Methodological issues and preliminary results on lexical patterns in Sl», International Journal of Translation 22 (1-2): 165-203.

Mariachiara RUSSO e Icíar ALONSO-ARAGUÁS

La interpretación en las organizaciones internacionales. Investigación, formación y práctica vol. 3-2, December 2017, 7-17

elSSN: 2444-1961

(C) Ediciones Universidad de Salamanca - CC BY-NC-ND 